\title{
A double blind study of single dose azithromycin and doxycycline in the treatment of chlamydial urethritis in males
}

\author{
A Nilsen, A Halsos, A Johansen, E Hansen, E Tørud, D Moseng, G Ånestad, \\ G Størvold
}

\begin{abstract}
Objective-To compare the efficacy and safety of azithromycin and doxycycline in the treatment of males with uncomplicated urethritis caused by chlamydia trachomatis.
\end{abstract}

Design-A multicentre, double-blind, randomised treatment study.

Subjects-130 male outpatients with clinical signs and symptoms of urethritis.

Setting-STD clinics at four Norwegian University Hospitals.

Methods-Patients were randomly allocated to $1000 \mathrm{mg}$ azithromycin as single dose or doxycycline $100 \mathrm{mg}$ twice daily for 7 days. Clinical, bacteriological and safety assessments were made at entry and after 1 and 2 weeks. Safety data were also repeated after 4 weeks.

Results-Demographic data were similar in both groups. At the week 1 assessment bacteriological eradication was achieved in 44 of 44 evaluable azithromycintreated patients and in $\mathbf{4 2}$ of $\mathbf{4 2}$ in the doxycycline group. At the week 2 assessment the corresponding figures were 35 of 35 and 34 of 34 respectively.

Conclusion-Azithromycin $1000 \mathrm{mg}$ single dose was as effective as doxycycline $\mathbf{1 0 0}$ mg twice daily for 7 days in male patients with chlamydial urethritis.

Dermatovenerology

University of Bergen,

Bergen, Norway

A Nilsen

Department of

Dermatovenerology,

University of

Trondheim,

Trondheim, Norway

E Hansen

E Torud

Department of

Dermatovenerology,

University of Tromse,

Tromse, Norway

D Moseng

The Municipal Health Centre, Oslo, Norway

A Halsos

A Johansen

The National Institute of Public Health, Oslo,

Norway

G Ánestad

Microbiological

Department, Ullevaal

Hospital, University of

Oslo, Oslo, Norway

G Størvold

Address for correspondence: Dr A Nilsen, Department of Dermatovenerology,

University of Bergen, 5021

Bergen, Norway.

Accepted for publication

31 March 1992 . Unike gonococcal infections, regimen availa infections.

Azithromycin has been shown to posess interesting pharmacokinetic properties. In contrast to low serum levels, high and sustained concentrations are found in a variety of different tissues including the urogenital tract. ${ }^{1}$ Furthermore, azithromycin has been shown to be highly active in vitro against several sexually transmittable agents including $\mathrm{CT}^{23}$ Especially because of the long tissue half life, this drug would seem to be an interesting candidate as a single-dose treatment regimen in chlamydial urethritis.

The objective of the present study was to compare the efficacy and safety of azithromycin and doxycycline in the treatment of males with uncomplicated urethritis caused by CT.

\section{Patients and methods}

Study population and design

The study was carried out at the outpatient clinics for sexually transmitted diseases of the University Hospitals in Bergen, Trondheim and Tromsø and at the Municipal Health Centre, Oslo.

Male patients, aged 18 years or more, presenting for diagnosis and treatment of acute urethritis, were invited to participate in the study. Entry criteria comprised clinical signs and symptoms of non-gonococcal urethritis. After giving informed consent, each patient underwent a general and genitourinary examination. Signs and symptoms of urethritis (discharge, burning, painful urination and other volunteered or observed symptoms) were categorised as mild, moderate or severe. A diagnosis of simple urethritis was made by demonstrating the presence of more than 10 polymorphonuclear leucocytes (PMNs) per high power field and the absence of gonococci on microscopic examination of Gram stained urethral smear. Blood and urine specimen were obtained for a panel of routine safety tests. Urethral swabs were collected for antigen test (CT) (one centre only) and for culture (CT and Neisseria gonorrhoea)(all centres). All tests were performed within 48 hours prior to the expected start of study treatment. Patients were re-evaluated 1 and 2 weeks after treatment. Blood and urine specimen were also repeated after 4 weeks. The patients were advised to observe sexual abstinence throughout their participation in the study.

In order of presentation patients who satisfied the entry criteria were assigned to oral regimens of either azithromycin or doxycycline according to a pre-determined randomisation list. Patients assigned to azithromycin were to take a single $1000 \mathrm{mg}$ dose as four capsules on the morning of day 1 . Additionally they were to 
Table 1 Reasons for exclusion from bacteriological and clinical evaluation

\begin{tabular}{|c|c|c|c|c|}
\hline & \multicolumn{4}{|c|}{ Treatment qroup } \\
\hline & \multicolumn{2}{|c|}{ Azithromycin } & \multicolumn{2}{|c|}{ Doxycycline } \\
\hline & Week 1 & Week 2 & Week 1 & Week 2 \\
\hline $\begin{array}{l}\text { REASON: } \\
\text { No baseline culture or no pathogen } \\
\text { isolated }\end{array}$ & 16 & 16 & 15 & 15 \\
\hline $\begin{array}{l}\text { Patient entered more than once } \\
\text { Concomitant antibiotic } \\
\text { No data at assessment } \\
\text { Unprotected sexual intercourse } \\
\text { before visit }\end{array}$ & $\begin{array}{l}1 \\
0 \\
2 \\
3\end{array}$ & $\begin{array}{l}1 \\
1 \\
6 \\
7\end{array}$ & $\begin{array}{l}0 \\
0 \\
6 \\
1\end{array}$ & $\begin{array}{l}0 \\
0 \\
6 \\
9\end{array}$ \\
\hline Total unevaluable & 22 & 31 & 22 & 30 \\
\hline
\end{tabular}

Table 2 Demographic data at entry

\begin{tabular}{lll}
\hline & \multicolumn{2}{l}{ Treatment qroup } \\
\cline { 2 - 3 } & Azithromycin & Doxycycline \\
\hline Age (years): & $25 \cdot 5$ & $26 \cdot 2$ \\
Mean & $18-42$ & $18-52$ \\
Range & $75 \cdot 4$ & $76 \cdot 1$ \\
Weight: & $53-93$ & $54-97$ \\
Mean & & \\
Range & 64 & 62 \\
Race: & 1 & 1 \\
White & 0 & 0 \\
Asian & 1 & \\
Black & & \\
Others & &
\end{tabular}

take one placebo capsule, followed 12 hours later by another placebo capsule. On day 2-7 inclusive they were to take one placebo capsule every 12 hours. Patients assigned to doxycycline were to take four placebo capsules and one doxycycline capsule $(100 \mathrm{mg}$ ) on the morning of day 1 , followed 12 hours later by another doxycycline capsule. The doxycycline treatment b.i.d. was continued for 7 days.

To be included in the study for evaluation of clinical and bacteriological effect, the presence of CT had to be verified by culture. The safety data were evaluated in all patients satisfying the entry criteria. Patients were excluded for the following reasons: evidence of gonorrhoea, concomitant treatment with another antimicrobial drug or with theophylline, ergotamine, warfarin or carbamezipine, known hypersensitivity to macrolides or tetracyclines, conditions likely to affect drug absorption, history or evidence of clinically significant haematological, renal, hepatic or cardiac disease, positive HBsAg or HIV test, known alcohol or drug abuse.

\section{Chlamydial culture}

Urethral samples were collected with a wiremounted cotton-tipped swab. After collection the swabs were transported to the laboratory in

Table 3 Clinical and bacteriological effect of azithromycin $1000 \mathrm{mg}$ single dose versus doxycyline $100 \mathrm{mg}$ bd for 7 days in uncomplicated male urethritis caused by Chlamydia trachomatis

\begin{tabular}{llllll}
\hline & \multicolumn{2}{l}{ Treatment qroup } & & \\
\cline { 2 - 3 } & \multicolumn{2}{l}{ Azithromycin } & & \multicolumn{2}{l}{ Doxycycline } \\
\cline { 2 - 3 } \cline { 5 - 6 } \cline { 5 - 6 } & Week 1 & Week 2 & & Week 1 & Week 2 \\
\hline Clinically cured & $28 / 44$ & $31 / 35$ & & $29 / 42$ & $32 / 34$ \\
& $(64 \%)$ & $(89 \%)$ & & $(69 \%)$ & $(94 \%)$ \\
Bacteriologically cured & $44 / 44$ & $35 / 35$ & & $42 / 42$ & $34 / 34$ \\
& $(100 \%)$ & $(100 \%)$ & & $(100 \%)$ & $(100 \%)$ \\
\hline
\end{tabular}

tubes with $1 \mathrm{ml} 0.2 \mathrm{~mol}$ sucrose-phosphate (2-SP) medium. The transport time was less than 24 hours for nearly all samples. Chlamydial culture was performed in cycloheximidetreated McCoy cells as described by Ripa and Mårdh. ${ }^{4}$ After 48-72 hours incubation the cells were fixed in methanol and stained with a commercial FlTC-conjugated monoclonal antibody to CT (Syva Company).

\section{Analyses}

All statistical tests were performed as twotailed tests. Between group differences were considered to be statistically significant if $p<$ 0.05 .

Efficacy: Only patients who completed the study according to the protocol were evaluated. All chlamydia isolates were assumed to be sensitive. Clinical response was classified as cured or not cured (failed) and was based on the patient's symptoms data. Clinical cure was defined as complete absence of symptoms and signs (excluding PMNs). In both the evaluation of clinical and bacteriological response, week 1 was defined as 6-12 days after the start of therapy, week 2 as 13-21 days. The distribution of clinical response of the two treatment groups were compared using the chi square test (with continuity correction), or Fischer's exact test for small samples (SAS procedure FREQ). The same procedure was used for comparison of bacteriological response rates. Safety: Any side effect occurring during therapy or within 35 days of the end of therapy were tabulated by severity, organ system and by the investigator's assessment of their relationship to the study drug.

\section{Results}

A total of 130 patients entered the study. Of the 66 patients in the azithromycin group, 22 patients were excluded from evaluation at week 1, most of them because of a chlamydia negative baseline culture (table 1). Additionally nine patients were excluded at week 2 , most of them because of unprotected sex before visit (table 1). In the doxycycline group the analogue exclusion figures are 22 and 8 respectively (table 1). Demographic data (table 2) were similar for both groups.

Clinical cure at week 1 was recorded in $28 / 44(64 \%)$ azithromycin-treated and in $29 / 42(69 \%)$ doxycycline-treated subjects. The corresponding figures for clinical cure at week 2 were $31 / 35(89 \%)$ and $32 / 34(94 \%)$ respectively (table 3 ). There was no significant difference $(p<0.05)$ between the two treatments with respect to clinical response. Eradication rates for $\mathrm{CT}$ at the week 1 evaluation were 44/44 and 42/42 in azithromycin- and doxycycline-treated patients respectively. The corresponding eradication rates at week 2 were $35 / 35$ and $34 / 34$ (table 3 ).

Treatment-related side effects were reported by $20 / 66(30 \%)$ azithromycin-treated and by $14 / 64(22 \%)$ doxycycline-treated patients, mostly mild to moderate gastrointestinal upset (table 4). Six patients in the azithromycin group and four in the doxycycline group 
Table 4 Reported side effects and laboratory abnormalities possibly related to the treatment

\begin{tabular}{lll}
\hline & \multicolumn{2}{l}{ Treatment qroup } \\
\cline { 2 - 3 } & Azithromycin & Doxycycline \\
\hline No. with side effect & $20 / 66(30 \%)$ & $14 / 64(22 \%)$ \\
Diarrhoea & 15 & 2 \\
Abdominal pain & 3 & 2 \\
Nausea & 2 & 4 \\
Increased sweating & 0 & 1 \\
General fatigue & 0 & 1 \\
Dyspepsia & 0 & 2 \\
Constipation & 0 & 1 \\
Flatulence & 0 & 1 \\
No. with abnormality & $6 / 64(9 \%)$ & $4 / 63(6 \%)$ \\
SGOT & $3(4 \cdot 6 \%)$ & $3(4 \cdot 7 \%)$ \\
SGPT & 0 & $2(3 \cdot 1 \%)$ \\
LDH & 1 & 0 \\
Bilirubin & 1 & 0 \\
Alkaline phosphatase & 0 & 1 \\
WBC & 1 & 1 \\
RBC & 1 & 0 \\
Differential count & 1 & 1 \\
\hline (more than 1 laboratory abnormality in each patient)
\end{tabular}

showed minor laboratory abnormalities possibly related to the treatment, mainly transient and mild increases in liver enzymes (table 4). There were no significant differences between the treatment groups.

\section{Discussion}

A few clinical studies have indicated that single dose or 2-3 days regimen of azithromycin is effective in uncomplicated genital chlamydial infection. $^{5-8}$ Some of these studies were not blinded and the patient populations were rather heterogeneous. Steingrimsson et al found a cure rate of $96 \%$ (113 chlamydia positive patients) with different azithromycin regimens inclusive $1000 \mathrm{mg}$ single dose in a blinded study. ${ }^{5}$ In a smaller study Lassus reported bacteriological eradication in 13/13 chlamydia positive patients. ${ }^{6}$ Whatley et al found a slightly better (but not significant) effect of $500 \mathrm{mg}$ azithromycin given as a single dose followed for 2 days by $250 \mathrm{mg}$ daily as compared with $1000 \mathrm{mg}$ single dose with respect to absence of symptoms and reduction in urethral PMNs. ${ }^{7}$ In a multicentre study comprising 85 male and 152 female patients Martin et al reported similar cure rates for azithromycin in uncomplicated genital chlamydial infection. ${ }^{8}$ Our study confirms these results. Azithromycin $1000 \mathrm{mg}$ given as a single dose is equally effective as doxycycline $100 \mathrm{mg}$ bd. for 7 days in uncomplicated chlamydial urethritis in males. This is the first drug that seems to be effective in single dose regimen in chlamydia infections. An obvious advantage of such treatment regimen is that the well-known problem of patient noncompliance could be eliminated.

It might be argued that the follow-up period should have been extended in our study. However, Martin et al $^{8}$ found similar bacteriological eradication rates at week 4 as compared with week 1 and 2 . The discussion of a possible "latency" after short treatment courses, with later relapse of chlamydial infection remains unresolved. We must also bear in mind that absence of bacterial shedding is not necessarily identical with absence of disease. Further- more, not even chlamydial culture might be sensitive enough ${ }^{9}$ for a test of cure. Currently, therefore, assessment of therapeutic efficacy ideally should be judged by very long follow up studies. Such studies are desirable, but unfortunately very difficult to conduct. Improved diagnostic tests may alleviate these difficulties and thereby render it possible to get better efficacy data on traditional as well as new drugs for chlamydial infections.

In our study both treatment groups reported a rather high incidence of side effects (table 4); the difference between the two treatment groups was not statistically significant. As in other studies ${ }^{6-8}$ mild to moderate gastrointestinal side effects dominated. We believe that the study design itself may be partly responsible for the unexpected high incidence of reported side effects, as all patients were asked specifically at every visit if they had noticed anything that might be a drug related side effect. In this context, it is worth noticing that no patient wanted to withdraw from the study because of side effects. Furthermore, it is known that adverse effects generally tend to be more frequent when treating sexually transmitted diseases and urinary tract infections as compared with other infections. ${ }^{10}$ Additionally, the incidence of adverse effects also tends to be significantly higher in blinded than in open studies. ${ }^{10}$

So far, the results from this double-blind study demonstrate that azithromycin administered as a single $1000 \mathrm{mg}$ dose to male patients with urethritis caused by CT is as effective and safe as a standard course of doxycycline. Azithromycin might turn out to be the drug of choice in genital chlamydial infections.

The authors acknowledge the effort and support of $\mathrm{Mr}$ Trond Tennøe, Pfizer, Norway and Pfizer Central Research, Sandwich, UK. We also acknowledge the efforts of our colleagues Kristin Ryggen, Anders Todal and Kjell Midelfart, Dept. of Dermatovenerology, University of Trondheim, Norway.

1 Girard AE, Girard E, English AR, et al. Pharmacokinetic and in vivo studies with azithromycin (CP-62.993), a new
macrolide with an extended half-life and excellent tissue distribution. Antimicrob Agents Chemother 1987; 31:1948-54.

2 Slaney L, Plummer F, Ronald AR, Degagne P, Hoban D, Brunham RC. In-vitro activity of azithromycin, erythromycin, ciprofloxacin and norfloxacin against Neisseria gonorrhoeae, Haemophilus ducreyi and Chlamydia trachomatis. If Antimicrob Chemother 1990,25(Suppl A): 1-5.

3 Scieux C, Kappus EW, Quinn TC. In-vitro activity of azithromycin against Chlamydia trachomatis. $\mathcal{F}$ Antimicrob Chemother 1990;25(Suppl A):7-10.

4 Ripa KT, Mårdh P-A. Cultivation of Chlamydia trachomatis in cycloheximide-treated McCoy cells. $¥$ Clin Microbiol 1977;6:328-31.

5 Steingrimsson O, Olafsson JH, Thorarinsson H, Ryan RW, Johnson RB, Tilton RC. Azithromycin in the treatment of sexually transmitted disease. $f$ Antimicrob Chemother sexually transmitted disease.

6 Lassus A. Comparative studies of azithromycin in skin and soft-tissue infections and sexually transmitted infections by Neisseria and Chlamydia species. $\mathcal{f}$ Antimicrob Chemother 1990;25(Suppl A): 115-21.

7 Whatley JD, Thin RNT, Mumtaz G, Ridgway GL. Azithromycin vs doxycycline in the treatment of nongonococcal urethritis. International fournal of STD E AIDS. 1991;2:248-51.

8 Martin D. Azithromycin in the Treatment of Chlamydial Urethritis/Cervicitis: A Multicenter Comparative Trial. $N$ Engl $\mathcal{f}$ Med (In press)

9 Taylor-Robinson D, Thomas BJ. Laboratory techniques for the diagnosis of chlamydial infections. Genitourin Med 1991;67:256-66.

10 Rubinstein E. Side-Effects of Antibiotics. Abstracts (No. 523) of the 17th International Congress of Chemotherapy, Berlin. 23-28 fune, 1991. 